Article

\title{
Unique Slow Crack Growth Behavior of Isotactic Polypropylene: The Role of Shear Layer-Spherulites Layer Alternated Structure
}

\author{
Mingjin Liu, Jiaxu Luo, Jin Chen, Xueqin Gao, Qiang Fu and Jie Zhang *(1) \\ State Key Laboratory of Polymer Materials Engineering, College of Polymer Science and Engineering, \\ Sichuan University, Chengdu 610065, China; Mingjin1006@163.com (M.L.); luojiaxuzzz@163.com (J.L.); \\ chenjinsc1996@163.com (J.C.); gaoxueqin@scu.edu.cn (X.G.); qiangfu@scu.edu.cn (Q.F.) \\ * Correspondence: zhangjie@scu.edu.cn
}

Received: 10 October 2020; Accepted: 13 November 2020; Published: 20 November 2020

\begin{abstract}
With the development of polymer science, more attention is being paid to the longevity of polymer products. Slow crack growth (SCG), one of the most important factors that reveal the service life of the products, has been investigated widely in the past decades. Here, we manufactured an isotactic polypropylene (iPP) sample with a novel shear layer-spherulites layer alternated structure using multiflow vibration injection molding (MFVIM). However, the effect of the alternated structure on the SCG behavior has never been reported before. Surprisingly, the results showed that the resistivity of polymer to SCG can be enhanced remarkably due to the special alternated structure. Moreover, this sample shows unique slow crack propagation behavior in contrast to the sample with the same thickness of shear layer, presenting multiple microcracks in the spherulites layer, which can explain the reason of the resistivity improvement of polymer to SCG.
\end{abstract}

Keywords: iPP; alternated structure; slow crack growth

\section{Introduction}

Polymer materials possess great potential as tailoring either the chemical or physical structures of polymer in solid state at different scales can satisfy the requirement for a certain application. Controlling microstructures via adjusting processing conditions (temperature, shear rate, etc.) during processing is more efficient and operable in contrast to chemical modification. This is called the classical "structure-property" relationship, which is a guideline for polymer processing [1-3]. Hence, it is of vital importance to reveal the relationship between the microstructure and properties for academia and industry.

Polymer products often fall in a brittle behavior after they are long exposed to the service temperature and low stress, containing the formation of a craze at a point of stress concentration and the subsequent propagation and fracture of the materials [4-7]. This long-term brittle failure, so-called slow crack growth (SCG), determines the practical service life of materials. A large number of studies have been carried out to understand comprehensively this phenomenon and create the ways to improve considerably the polymer resistance to SCG. It has been confirmed in the previous literature that the molecular topological structure and morphologies, involving the content of tie molecules [8], the number of short-chain branches [9], the molecular weight and its distribution [10], and crystalline morphology [11], are closely related to resistance to SCG. For example, Brown studied the effect of molecular weight and branch density on the rate of SCG, and their results showed that a high molecular weight as well as short chain branch can enhance the resistance to SCG significantly $[10,12]$. Ludwig et al. discovered that polyethylene (PE) with a broad molecular weight distribution has 
superior long-term mechanical properties [13]. Meanwhile, many researches have proven that the formation of a shish-kebab structure and more perfect crystals can help to enhance the resistance of polymer to SCG to a large extent $[11,14,15]$.

It is well known that injection molding is one of the most important processing technologies for polymers. Nevertheless, the plastic parts prepared by conventional injection molding (CIM) only have a relatively low content of shish-kebab structure in the skin layer in contrast to the amounts of spherulites that constitute the core layer, resulting in the poor mechanical properties of products. To optimize their performance, the issue of increasing the number of shish-kebab structures via imposing an extra strong shear field on polymer melt, which would result in the "coil-stretch" transition of the molecular chains, has been stressed in the past decades [16,17]. A large amount of experiments have been carried out to demonstrate that some modified injection technologies, such as pressure vibration injection molding (PVIM) [18,19], oscillatory packing injection molding (OSIM) [20,21] and push-pull injection molding [22-24] could be applied to obtain self-reinforced parts involving a mass of shish-kebab structure. A novel multiflow vibration injection molding (MFVIM) technology based on PVIM has been proposed by our group in recent years, whose mechanism has been described in the previous papers $[18,25,26]$. We can not only prepare plastic parts including a high content of shish-kebab to induce self-reinforcement effect, but also parts with a shear layer-spherulites layer alternated structure by tuning the processing parameters such as injection pressure and interval time. It has been reported that the Izod impact strength could be remarkably enhanced for the products with the alternated structure $[27,28]$, and it could be further improved by thermal annealing at a suitable temperature for a certain time. The highest value climbed up to $90 \mathrm{KJ} / \mathrm{m}^{2}$ for isotactic polypropylene (iPP), while the value of the sample prepared by CIM is lower than $5 \mathrm{KJ} / \mathrm{m}^{2}$ [29]. However, the influence of the distribution of shear layer on the long-term mechanical properties has never been investigated.

iPP, one of the most important general polymeric materials, presents excellent performances and relatively low costs. It was chosen to be the material in the current work, and the injection molded parts with a distinctly different hierarchic structure were prepared by using CIM and MFVIM respectively. On the basis of the previous investigation, we try to understand the relationship between the long-term mechanical properties and unique shear layer-spherulites layer alternated structure for the first time. The microstructure and long-term mechanical properties were detected by polarized optical microscopy (POM), differential scanning calorimeter (DSC), scanning electron microscopy (SEM) and self-designed SCG device [11]. The results indicated that such a special morphology could enhance the resistance of iPP to SCG pronouncedly, and the mechanism was also proposed. This work provided a promising and easy way to alleviate the environmental problems to some extent by lengthening the service life of the products prepared by iPP.

\section{Experimental Section}

\subsection{Materials}

IPP (commercial grade T30S) with the density of $0.910 \mathrm{~g} / \mathrm{cm}^{3}$ and melt flow index (MFI) of $2.90 \mathrm{~g} / 10 \mathrm{~min}\left(230^{\circ} \mathrm{C}, 2.16 \mathrm{~kg}\right)$, was available from Lanzhou Petrochemical Company (Lanzhou, China). TX-10, as the surfactant, was applied to accelerate the process of slow crack growth.

\subsection{Samples Preparation}

In this work, conventional injection molding (CIM) and multiflow vibration injection molding (MFVIM) samples were prepared by adjusting the pressure and interval time during the packing stage, and they were labeled as CIM, $V_{1}, V_{2}$ respectively. $V_{1}$ represents the sample with simply increased thickness of shear layer, while $V_{2}$ is the one with unique shear layer-spherulites layer alternated structure. It should be noted that both of them almost have the same thickness of shear layer. The temperature profile from hopper to nozzle was $160,180,190,200$, and $200{ }^{\circ} \mathrm{C}$, respectively, and the mold temperature was fixed at $50^{\circ} \mathrm{C}$. Some molding parameters are listed in Table 1 . 
Table 1. Molding parameters of vibration injection molding process.

\begin{tabular}{cccc}
\hline & CIM & V1 & V2 \\
\hline Injection pressure (MPa) & 40 & 40 & 40 \\
Packing pressure (MPa) & 30 & 30 & 30 \\
Vibration pressure (MPa) & & 60 & 100 \\
Interval time(s) & & $1 / 4 / 8$ & $10 / 5$ \\
\hline
\end{tabular}

After the samples were injection molded, dumbbell bars were cut from the same position for all specimens to investigate the long-term mechanical properties, which were $4 \mathrm{~mm}$ width and $3 \mathrm{~mm}$ thickness. We made a notch in the middle location for all the samples (shown in Figure 1) by a blade. The crack tips were sharp and the initial crack lengths were measured by vernier caliper. It should be noted that we tested three times for each kind of sample to avoid small differences in the initial crack length that may produce different behaviors.

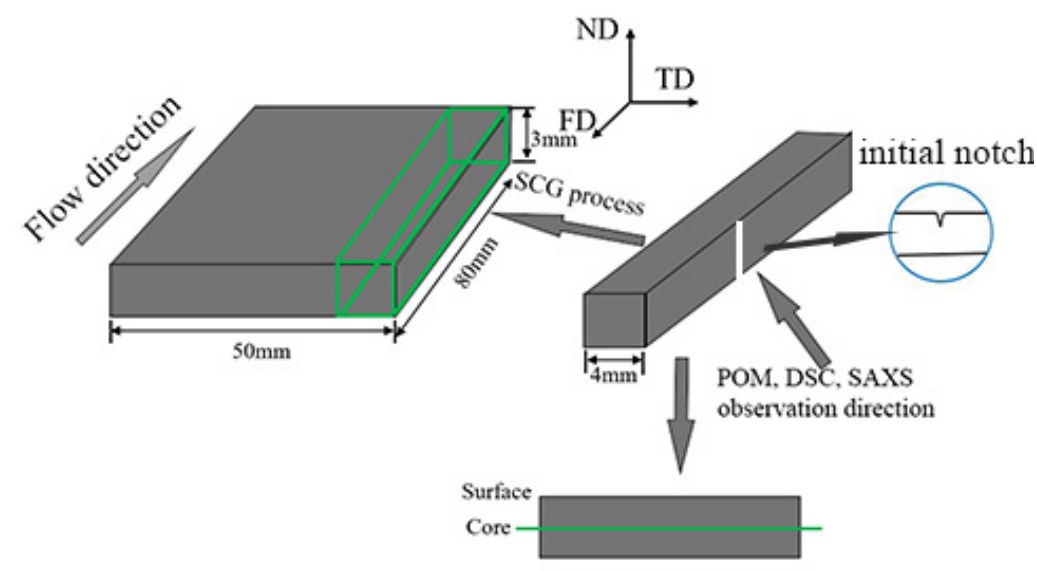

Figure 1. Schematic diagram of sample prepared for characterizations. FD, flow direction; TD, transverse direction; ND, normal direction.

\subsection{Polarized Optical Microscopy (POM)}

Thin slices with thickness of $30 \mu \mathrm{m}$ were cut from different samples at the same position by a microtome. Then the slices were observed by a DX-1 (Jiang Xi Phoenix Optical Co., Shangrao, China) microscope connected with a Canon 500D digital camera (Canon, Tokyo, Japan), and the observation direction for POM was parallel to transverse direction (shown in Figure 1).

\subsection{Differential Scanning Calorimeter (DSC)}

A DSC (TA Q200) device was used to analyze the thermal behavior of different samples. All measurements were carried out under dry nitrogen atmosphere. Specimens about 3-8 mg were heated from 80 to $200{ }^{\circ} \mathrm{C}$ with a heating rate of $10^{\circ} \mathrm{C} / \mathrm{min}$. The following equation was utilized for calculating the total crystallinity, $\mathrm{X}_{c}$, of each sample:

$$
X_{c}=\frac{\Delta H_{m}}{\Delta H_{m}^{o}}
$$

where $\Delta H_{m}$ represents the measured value of the enthalpy of fusion and $\Delta H_{m}^{o}$ manifests the fusion enthalpy of completely crystallized iPP. Here, the value of $\Delta H_{m}^{o}$ was selected as $207 \mathrm{~J} / \mathrm{g}$.

\subsection{Synchrotron Two-Dimensional X-ray Measurements}

2D small-angle X-ray scattering (2D-SAXS) were conducted on the BL16B1 beamline in Shanghai Synchrotron Radiation Facility (SSRF), Shanghai, China. The dimensions of the rectangle- 
shaped beam were $0.5 \times 0.8 \mathrm{~mm}^{2}$, and the wavelength of light was $0.124 \mathrm{~nm}$. The sample-to-detector distance was $1900 \mathrm{~mm}$ for SAXS.

The long period $\left(L_{p}\right)$ of SAXS results can be calculated by Bragg's law as follows:

$$
L_{p}=\frac{2 \pi}{q_{\max }}
$$

where $q_{\max }$ is the peak position of the 1D-SAXS intensity profile. The crystallite thickness $\left(L_{\mathcal{c}}\right)$ is calculated as long period multiplied by the crystallinity. The thickness of the amorphous phase $\left(L_{a}\right)$ is calculated by $L_{p}-L_{c}$.

\subsection{Scanning Electron Microscopy (SEM)}

A JEOL field emission scanning electron microscope (model JSM7500F, Tokyo, Japan) was employed to carefully observe the fracture morphology of different samples after suffering the slow crack growth process. Before observation, the specimens were gold sputtered.

\subsection{Slow Crack Growth Process (SCG)}

The slow crack growth experiment of the various samples was conducted on the self-designed SCG device. $10 \%$ TX-10, as the surfactant, was used to accelerate this process and the test temperature was maintained at $50{ }^{\circ} \mathrm{C}$. The initial stress in the experiment was different for sample CIM (5.9 MPa), $\mathrm{V}_{1}(7.2 \mathrm{MPa})$ and $\mathrm{V}_{2}(7.2 \mathrm{MPa})$ due to the variation of tensile strength among the samples. We used spring scale to correct the real initial stress loading on the samples. Due to the addition of TX-10, the induction period for the crack was considerably shorter than it would be under the normal conditions, but the process of SCG was the same, which could uncover the relationship between the microstructure and resistance to slow crack propagation. For the accuracy of the experiment, at least three specimens were tested to estimate the slow crack growth process for each sample.

\section{Results and Discussion}

\subsection{Crystalline Structure}

The sample prepared by CIM manifests a typical skin-core structure, which is in agreement with the previous papers published before [2], while sample $V_{1}$ and $V_{2}$ show special microstructure observed from Figure 2. It should be noted here that the thickness of shear layer of samples $V_{1}$ and $V_{2}$ is almost the same (about $55 \%$ ), indicating the same content of shish-kebab for these two samples, because it was speculated that the shear layer consisted fully of shish-kebab structure. More detailed information about the microstructure of all the samples, such as crystalline morphologies, orientation degree, the content of $\beta$ crystal, have been reported in our previous literature [29]. Here, we can reasonably conclude that manipulated microstructures with different shear layer distributions could be obtained for the parts through MFVIM.

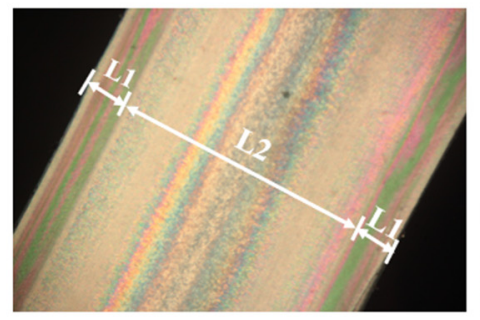

CIM

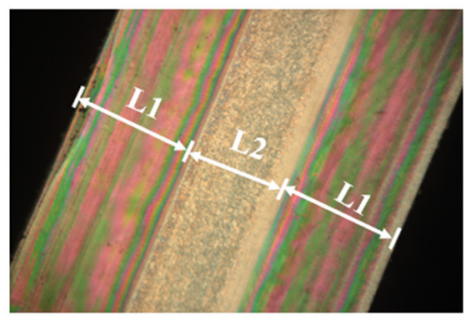

V1

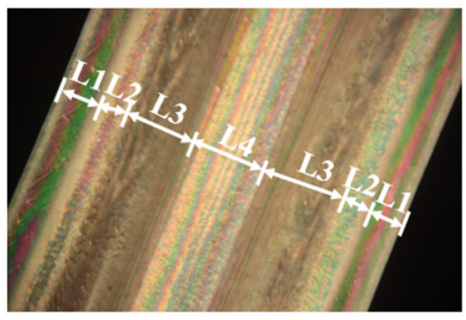

V2

Figure 2. Polarized optical microscopy (POM) photographs of different specimens. L1 and L3 represent the shear layer, L2 and L4 are the spherulites layer. 
The melting behavior of samples was estimated by calculating the melting temperature and crystallinity. We obtained these two parameters from shear and spherulites layer respectively resulting from the extremely different structure for shish-kebab and spherulites. It should be noted that just selected data was presented here, because the layers containing the same crystalline morphologies showed the similar value for each sample. For example, the value of $T_{m}$ and $X_{c}$ of layer L2 and L4 for sample $V_{2}$ is almost the same. As illustrated in Figure 3, these three samples presented the analogous melting behavior in the same kind of layer regardless of the shear layer or spherulites layer. The melting temperature and crystallinity of different layers are collected in Table 2. Theoretically speaking, the melting point of shish-kebab should be higher than that of spherulites because the former is regarded as a more thermal stable state. It is noticeable that the melting temperature scarcely changes in spite of the completely varied microstructure for shish-kebab and spherulites.

(a)

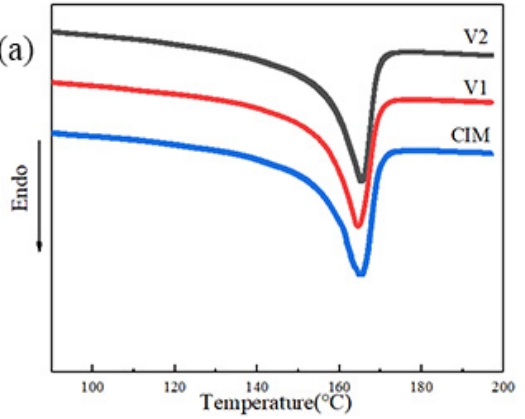

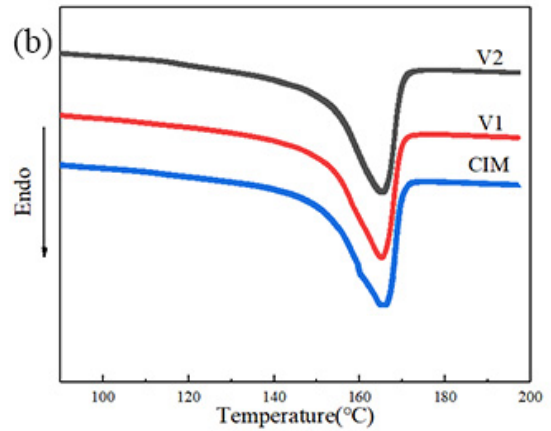

Figure 3. Differential scanning calorimeter (DSC) melting curves of different layers of samples, (a) shear layer, (b) spherulites layer.

Table 2. Melting temperature and crystallinity of different layers of samples.

\begin{tabular}{cccc}
\hline Sample & $\mathbf{C I M}$ & $\mathbf{V}_{\mathbf{1}}$ & $\mathbf{V}_{\mathbf{2}}$ \\
\hline$T_{m}$ of shear layer $\left({ }^{\circ} \mathrm{C}\right)$ & 165.29 & 164.57 & 165.21 \\
$T_{m}$ of spherulites layer $\left({ }^{\circ} \mathrm{C}\right)$ & 165.14 & 165.11 & 165.55 \\
$X_{c}$ of shear layer $(\%)$ & 44.4 & 44.6 & 45.3 \\
$X_{c}$ of spherulites layer $(\%)$ & 44.5 & 42.7 & 43.2 \\
\hline
\end{tabular}

For the sake of obtaining more information on crystalline structure, SAXS experiment was carried out. The selected results were shown in Figure 4 and the long period and the lamellae thickness of samples were collected in Table 3. From the results presented here, we can clearly know that the long period just changes a little for all the samples, regardless of shish-kebab or spherulites. Specifically, the value of $L_{p}$ of shish-kebab for sample $V_{1}$ and $V_{2}$ is 31.67 and 31.81 respectively, which is only slightly higher than that of specimen CIM $(28.98 \mathrm{~nm})$. Meanwhile, $L_{p}$ of spherulites remains almost the same. Coupling the DSC with SAXS results, the crystalline thickness $L_{\mathcal{c}}$ could be calculated directly (shown in Table 3). It shows that the crystalline size of these three samples, regardless of shish-kebab or spherulites, is relatively similar, indicated by almost the same value of crystalline thickness.
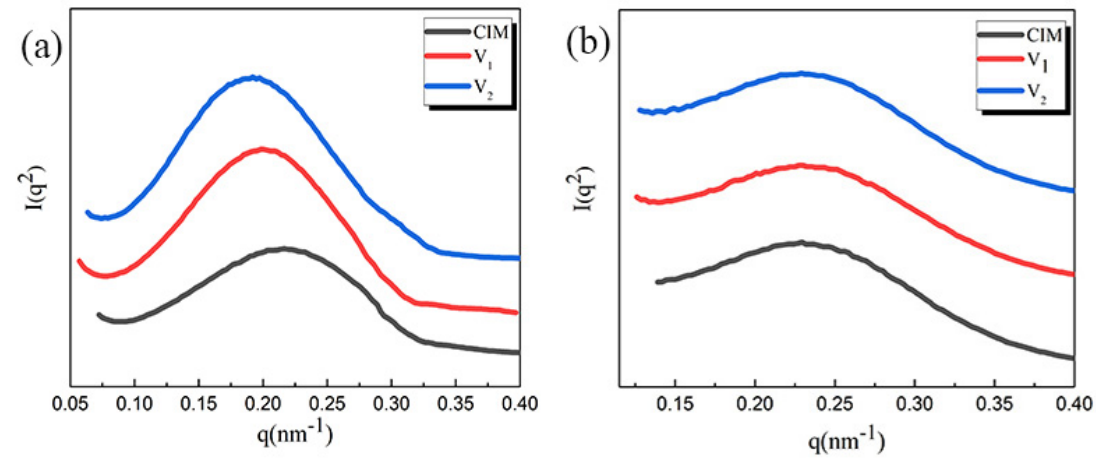

Figure 4. Corresponding intensity profiles of 1D-SAXS for different samples as a function of the scattering vector (q): (a) shear layer; (b) spherulites layer. 
Table 3. Value of long period and crystalline size of different layers of samples.

\begin{tabular}{cccc}
\hline Sample & $\mathbf{C I M}$ & $\mathbf{V}_{\mathbf{1}}$ & $\mathbf{V}_{\mathbf{2}}$ \\
\hline$L_{p}$ of shish-kebab $(\mathrm{nm})$ & 28.98 & 31.67 & 31.81 \\
$L_{p}$ of spherulites $(\mathrm{nm})$ & 27.36 & 27.29 & 27.41 \\
$L_{c}$ of shish-kebab $(\mathrm{nm})$ & 12.87 & 14.12 & 14.41 \\
$L_{c}$ of spherulites $(\mathrm{nm})$ & 12.18 & 11.65 & 11.84 \\
\hline
\end{tabular}

As discussed above, it can be well known that the molecular topological and crystalline structure associated with the capacity for resisting slow crack propagation, such as the content of shish-kebab, the distance between the lamellae and the perfection of the lamellae, are completely similar for sample $V_{1}$ and $V_{2}$ regarding shear and spherulites layer. Hence, the difference between these two samples is just the distribution of shear layer or spherulites layer. Next, we would study the role of alternated structure on the slow crack growth behavior to provide a practical application prospects for sample with such a novel structure. So, the results and discussion are focused on samples $V_{1}$ and $V_{2}$ in the later section.

\subsection{Slow Crack Growth Process}

In the plastic parts applications, SCG is one of principal failure modes, which is initiated by a defect or stress concentration, containing the formation of a craze at a point of stress concentration, the subsequent crack propagation and fracture of the materials. The SCG process would not take extremely long time to complete under the suitable conditions for selected samples. The initial stress we selected for sample CIM was $5.9 \mathrm{MPa}$, and it was $7.2 \mathrm{MPa}$ for both sample $\mathrm{V}_{1}$ and $\mathrm{V}_{2}$. The reason why we chose two different initial stresses was the obvious difference of tensile strength between sample CIM and the other two specimens. Further, it has been confirmed that the value of yield strength of samples $V_{1}$ and $V_{2}$ was similar [11], so we used the identical initial stress to investigate the evolution of slow crack growth for these two samples.

Figure 5 shows the patterns of the evolution of crack propagation of samples under a low initial stress for different times at $50{ }^{\circ} \mathrm{C}$. Note that there are some dots in this figure, which is induced by some impurities or bubbles. We can clearly observe that the evolution of the slow crack growth for sample CIM is relatively rapid compared to the other two specimens, the sample completely fractures only within $507 \mathrm{~min}$, which indicates the poor capacity of the resistivity of the material to the slow crack propagation behavior. The phenomenon could be explained by the low content of shish-kebab in the sample manufactured by conventional injection molding. Compared with sample $\mathrm{CIM}$, the total fracture time of sample $\mathrm{V}_{1}$ climbs from $507 \mathrm{~min}$ to $1380 \mathrm{~min}$ originating from the high content of shish-kebab structure induced by the extra imposed shear field, which is consistent with the previous work [11]. These two samples underwent a complete fracture process under the experimental conditions used in this work. The difference of fracture process in the initial stage between samples $V_{1}$ and $V_{2}$ is considerably slight as illustrated in Figure 5. Surprisingly, the fracture behavior of sample $V_{2}$ (shown in Figure 5) at the later stage is totally different from $V_{1}$, showing multiple microcracks except for the original notch in this sample, indicated by the presence of stress white region, which is absent in the sample CIM and $V_{1}$. The loading that the initial notch suffered would be shared through the formation of microcracks, leading to the suppression of slow crack growth. The reason why the stress white region formed will be discussed in a later section. 

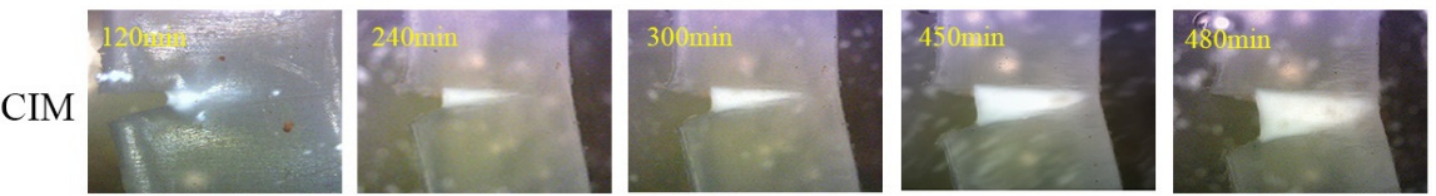

V1
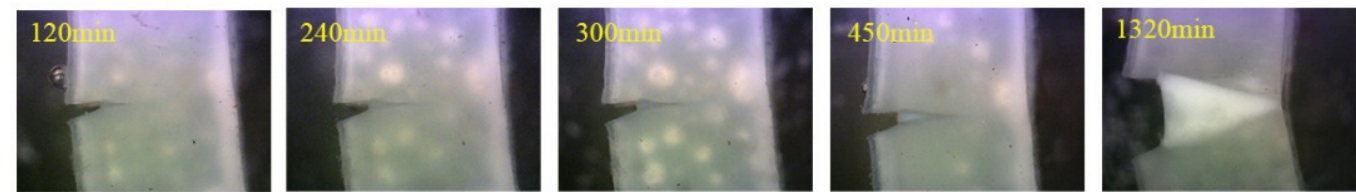

V2
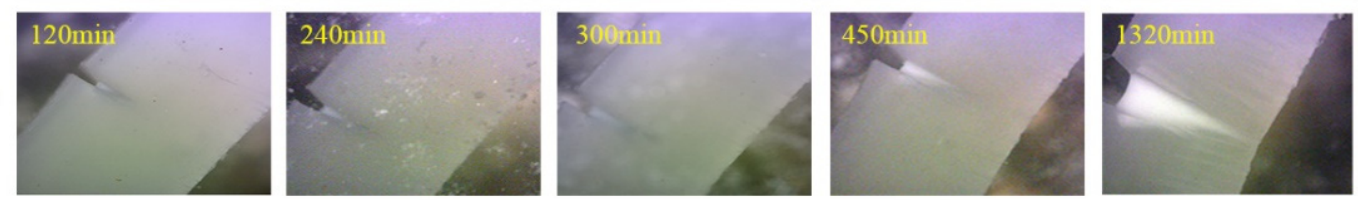

Figure 5. The patterns of the crack growth of specimens under a low initial stress for different times at $50^{\circ} \mathrm{C}$.

Figure 6 manifests the patterns of crack propagation of sample $V_{1}$ and $V_{2}$ at the later stage to furtherly reveal the different fracture process. It can be well observed that stress white region is distinctly obvious for sample $V_{2}$ when the fracture time is over $24 \mathrm{~h}$, but not for sample $V_{1}$. Sample $V_{1}$ would undergo the complete fracture process under the low initial stress without microcracks formed. Here, it should be noted that the deformation of sample $V_{2}$ along the tensile direction had reached the measuring limit of SCG device after subjected to the low stress for $2400 \mathrm{~min}$, originating from the formation of numerous microcracks at the later stage. The reason why there are not obvious microcracks could be observed at the early stage may be attributed to the long induction period that defects propagate into microcracks under the low stress. We could speculate that the microcracks formed during the slow crack growth process can prominently decrease the stress at the initial crack tip. That is to say, the stress loaded on the sample can be shared with a large number of microcracks besides the initial notch, which enhances the resistivity of this sample to the SCG process to a large extent. It is of practical or scientific significance for the development of polymer science and engineering. Hence, we can have a reasonable vision that the sample with such a novel shear layer-spherulites layer alternated structure could lengthen the service life of the products under some conditions.
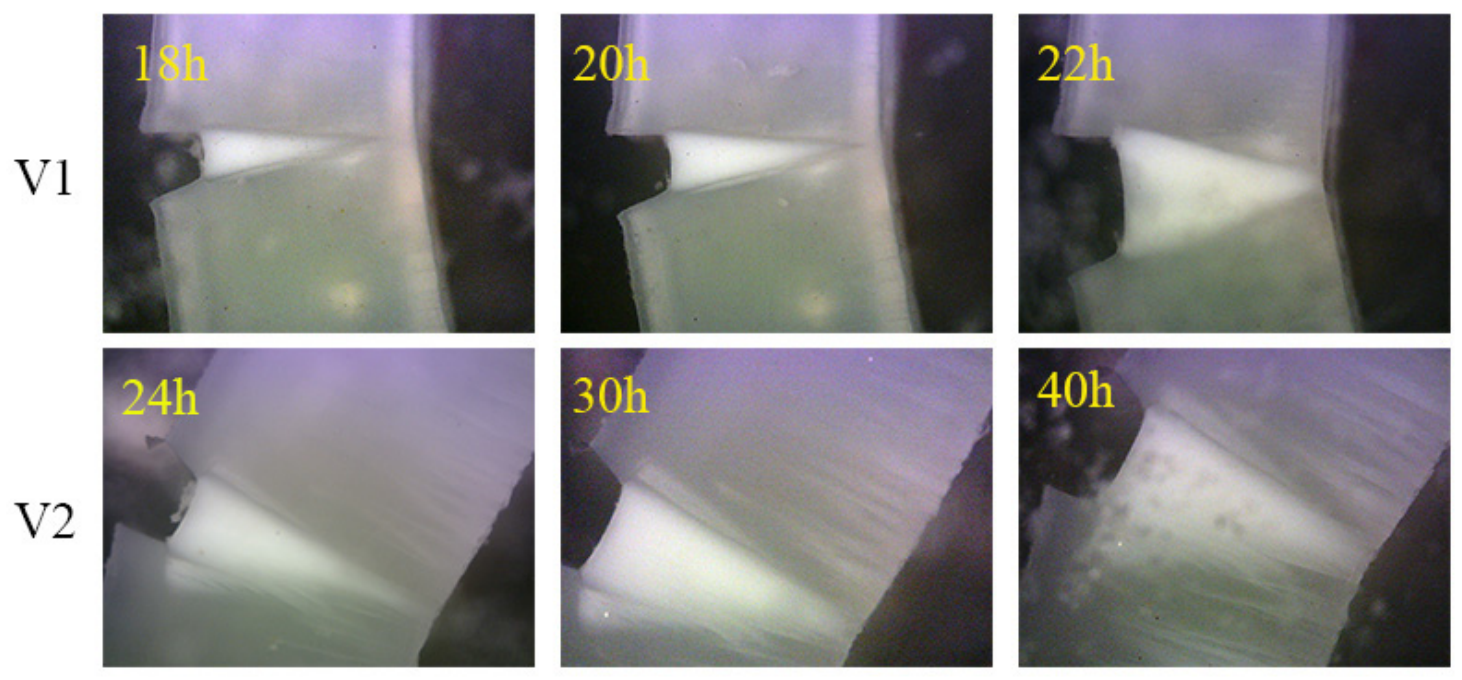

Figure 6. The patterns of crack propagation of sample at the later stage. 
Figure 7 quantitatively characterizes the crack growth process by introducing the crack length versus time curves. It could be well observed that sample CIM shows rapid crack propagation, indicated by fracture within a short time. As for the other two samples, they possess similar fracture process in the early fracture stage, but the crack growth behavior is extremely different during the later fracture stage, the crack propagation of sample $V_{2}$ was suppressed showing the independence of time during the later stage, which is consistent with the result of Figures 5 and 6.

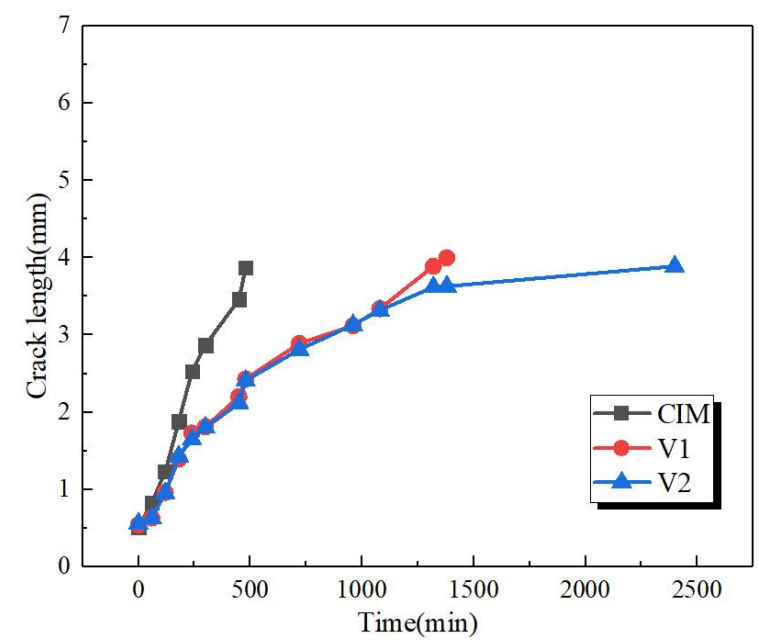

Figure 7. The crack length versus time of all the samples during the process of slow crack growth (SCG).

In order to get more detailed information about the fracture morphology of sample $V_{1}$ and $V_{2}$ after the SCG process, Figure 8 presents its SEM images, and the observation direction of SEM is the transverse direction as shown in Figure 1 . The extremely different morphologies for sample $V_{1}$ and $V_{2}$ can be well observed. The sample $\mathrm{V}_{1}$ (shown in Figure 8a) manifested relative smooth surface with the absence of microcracks. It should be noted clearly that the sample $V_{2}$ does not fracture completely after suffered the SCG process for a very long time, and there are many stress white regions throughout the specimen. Corresponding to the POM photograph presented in Figure 2, it can be known from Figure $8 \mathbf{b}$ that microcracks formed in the spherulites layer but not the shear layer due to the inferior properties for spherulites. Figure $8\left(b_{1}, b_{2}\right)$ show the magnification of morphologies at corresponding locations in Figure 8b. A large amount of microcracks can be observed more clearly, which further confirms the existence of microcracks in this sample. Here, the reason why microcracks formed only in the spherulites layer for sample $V_{2}$ will be discussed later to provide a promising way to manufacture robust plastic parts to meet the requirements of industry.
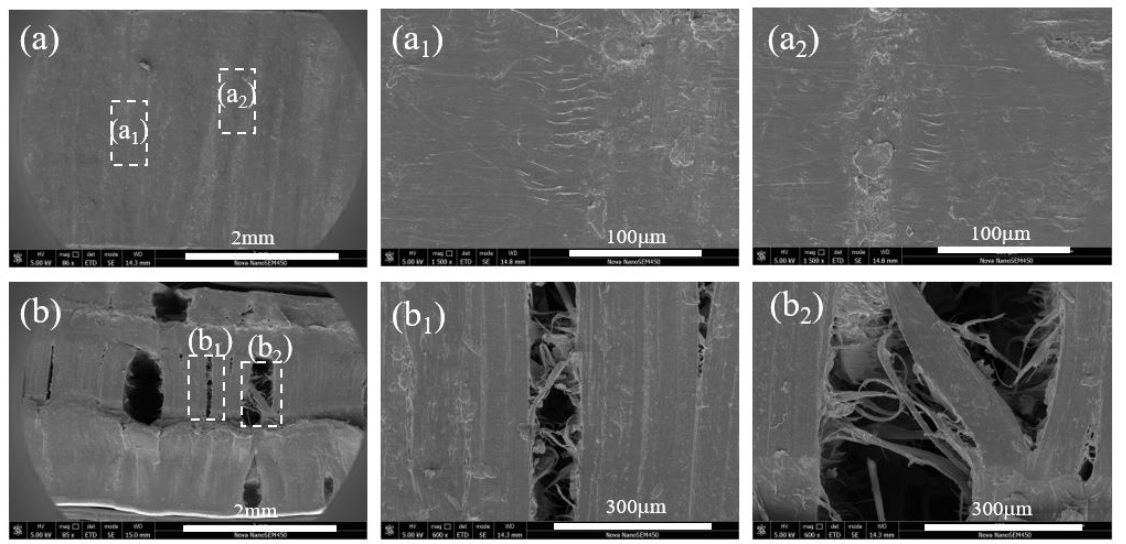

Figure 8. The SEM images of morphology of sample after suffering SCG process. (a): $V_{1},(\mathbf{b}): V_{2}$. $\left(\mathbf{a}_{1}\right),\left(\mathbf{a}_{2}\right)$ and $\left(\mathbf{b}_{1}\right),\left(\mathbf{b}_{2}\right)$ are the corresponding magnified images in $(\mathbf{a})$ and $(\mathbf{b})$ respectively. The observation direction of SEM is the transverse direction (TD) shown in Figure 1. 
The SEM images A, B and C, shown in Figure 9, represent the morphology of the whole fracture surface for the samples CIM, $V_{1}$ and $V_{2}$ respectively. While the other two columns, such as $A_{1}$ and $A_{2}$, manifest the magnification of morphologies at the corresponding locations marked in the first column. It is obvious that the fracture surface of sample CIM is relatively smooth in the forepart in contrast to the rear part. Each part represents the fracture process at a different time. Brittle fracture (Figure 9A1) showing smooth morphology appears in the early stage of the crack growth. Then plastic tensile deformation appears, and it evolves plastic failure in the posterior stage leading to the coarse fracture surface as shown in Figure 9A2. Compared to sample CIM, the fracture morphology of $V_{1}$ presents two totally different regions, which is associated with crystalline morphology. It is clear that the fracture in the shear layer (Figure 9B1) presents brittle fracture due to the high content of shish-kebab. However, in the core regime, the whole region manifests a rough morphology as shown in Figure 9B2, resulting from the inferior resistivity capacity of spherulites and the high initial stress or temperature. As for the sample $V_{2}$, shown in Figure 9C, it should be noted that this specimen after suffering SCG process was cut along the direction of the original notch by a blade to observe the surface morphology because it was not broken during the whole fracture process. The extremely smooth surface is shear layer (Figure 9C1), which is a signal of no defects formed in this layer. While plastic tensile deformation appears in the spherulites layer, indicated by the presence of plastic deformation observed in Figure 9C2, and plastic deformation induces a large content of microcracks in the spherulites layer. It could be known that the results concerning the sample $\mathrm{V}_{2}$ are in agreement with those manifested in Figure 8. That is, multiple microcracks form in the spherulites layer after the slow crack growth process is complete.
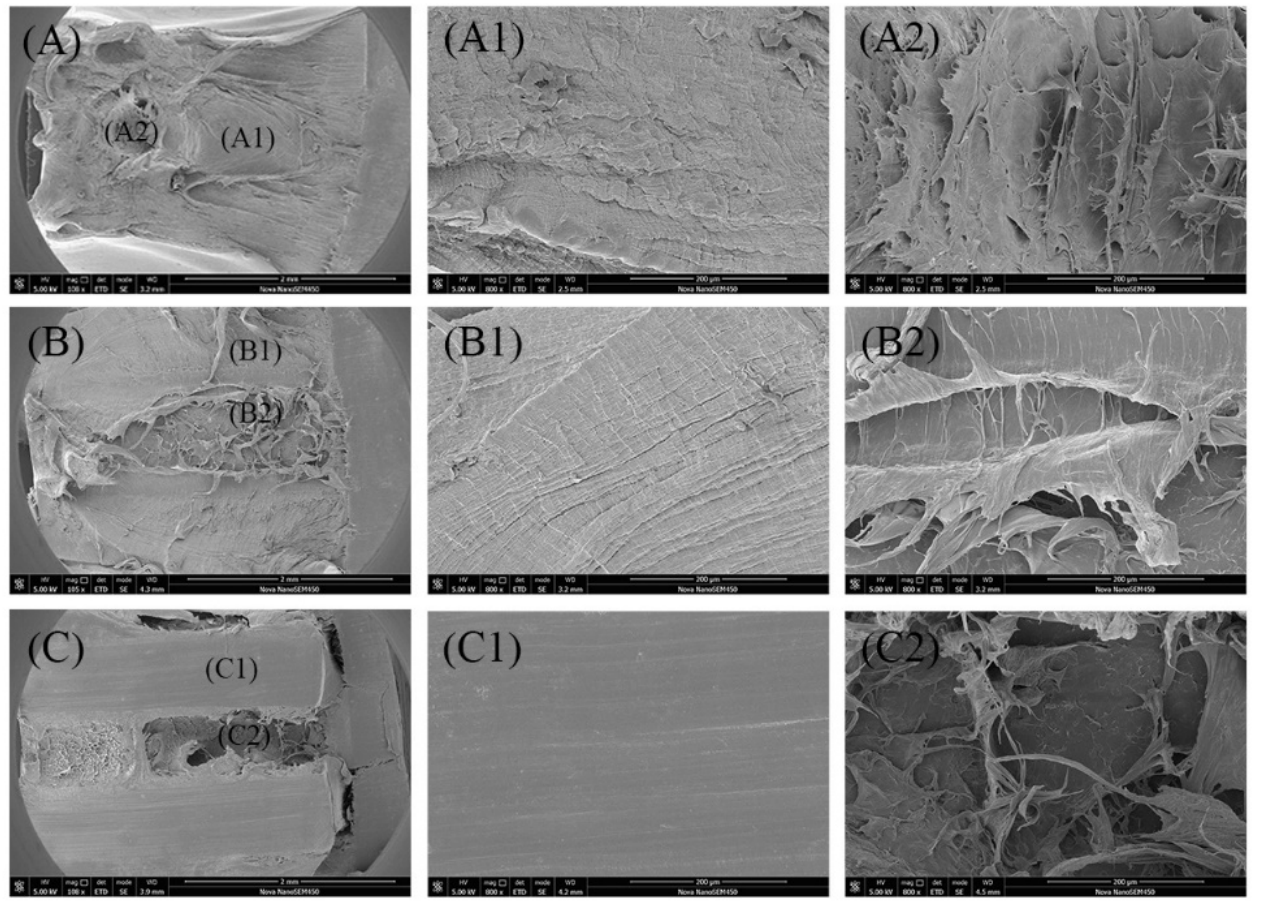

Figure 9. SEM images of the fracture morphology of specimens, the crack propagation direction is from right to left, (A) CIM; (B) $\mathrm{V}_{1}$; (C) $\mathrm{V}_{2}$. (A1), (A2), (B1), (B2) and (C1), (C2) are the corresponding magnified images in $(\mathbf{A}),(\mathbf{B})$ and $(\mathbf{C})$, respectively.

The failure process of sample with the special alternated structure has been discussed above, and such unique phenomenon has never been reported for virgin polymers in the previous research. As demonstrated in Figures 6 and 8, a large number of microcracks are formed in the spherulites layer for sample $V_{2}$, which presents extremely different fracture process (shown in Figure 10) in contrast to sample $\mathrm{V}_{1}$ and CIM. This is so-called dispersion damage mechanism, which usually 
occurs in fiber reinforced materials under the stress, resulting in the large consumption of energy during the fracture process. That is to say, the multiple microcracks can share the stress loaded on the sample with initial notch, so the crack propagation process would be suppressed significantly. It can be stated that local stress concentrations induced by microvoids could initiate yielding in the amorphous region firstly, this is followed by the fragmentation of crystalline lamellae and partial chains unfolding. Then, a fibrillar structure is generated when a cluster of uniaxially oriented molecules and intermediary voids are developed. Thus, the damage region grows into a crazing zone. After the craze is initiated, a crack slowly evolves when the fibrils fail under stress due to disentanglement and break-up of interlamellar tie molecules. The reasons for the formation of multiple microcracks in the spherulites of sample $V_{2}$ may be summarized as follows: the interface adhesion strength between spherulites and shear layer is poor when the thermal annealing process is absent, leading to the easier formation of defects at the interface. So, many defects would occur under the conditions used in this work. These defects would propagate into microcracks, and microcracks are more easily formed in the spherulites layer resulting from the inferior capacity for resisting load. These factors cause the dispersion damage phenomenon for sample $\mathrm{V}_{2}$. However, some studies are still required for further accurately understanding this unique phenomenon.

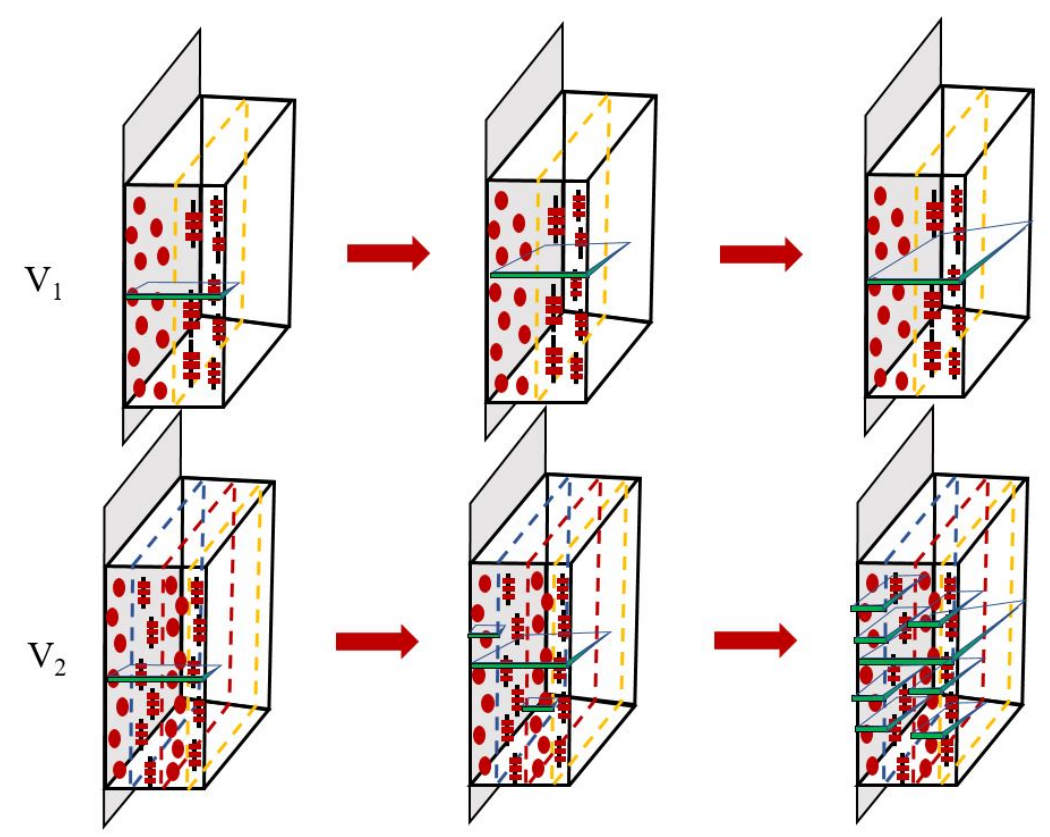

Figure 10. Schematic drawing of failure process of sample $V_{1}$ and $V_{2}$ under low initial load.

\section{Conclusions}

In the current work, samples with extremely different microstructures were successfully prepared by using CIM and MFVIM, respectively. The crystalline structure and the slow crack growth behavior were investigated. The DSC and 2D-SAXS results indicated that the crystalline structure associated with the capacity for resisting slow crack propagation, such as the content of shish-kebab, the distance between the lamellae, and the perfection of the lamellae, scarcely varies for sample $V_{1}$ and $V_{2}$. Compared with sample CIM, the total fracture time for samples $V_{1}$ and $V_{2}$ are remarkably enhanced due to the large number of shish-kebabs originating from the extra imposed shear field during the packing stage. Interestingly, the slow crack growth behavior of sample $\mathrm{V}_{2}$ not only manifested the improvement of the complete fracture time, but also the unique crack propagation behavior, showing that multiple microcracks formed in the spherulites layer. This is so-called dispersion damage, forming a large number of microcracks, which results in the reduction of load for the initial notch, and can further enhance the service life of polymer products through the formation of multiple 
microcracks. The results of this work indicated the practical application prospect of a sample with a shear layer-spherulites layer alternated structure in the near future.

Author Contributions: Data curation, M.L.; Funding acquisition, J.Z.; Methodology, M.L.; Project administration, J.C. and J.Z.; Supervision, X.G., Q.F. and J.Z.; Validation, J.L. and J.C.; Writing-original draft, M.L. All authors have read and agreed to the published version of the manuscript.

Funding: This research was funded by the National Natural Science Foundation of China grant number [NO. 21627804] and State Key Laboratory of Polymer Materials Engineering grant number [No. sklpme2019-2-18].

Acknowledgments: The authors sincerely acknowledge the technical support of the Shanghai Synchrotron Radiation Facility (SSRF, Shanghai, China) for help with X-ray measurements.

Conflicts of Interest: The authors declare no conflict of interest.

\section{References}

1. Housmans, J.-W.; Gahleitner, M.; Peters, G.W.M.; Meijer, H.E. Structure-property Relations in Molded, Nucleated Isotactic Polypropylene. Polymers 2009, 50, 2304-2319. [CrossRef]

2. Wang, Y.; Hou, F.; Mi, D.; Zhou, M.; Jiang, Y.; Zhang, J. Self-Reinforcement of Polypropylene Lid-Shaped Samples Induced by Increasing Shish-Kebab Content: Practical Application of Vibration Injection Technology. Ind. Eng. Chem. Res. 2018, 57, 8620-8629. [CrossRef]

3. Kantz, M.R.; Newman, H.D.; Stigale, F.H. The Skin-Core Morphology and structure-property Relationships in Injection-Molded Polypropylene. J. Appl. Polym. Sci. 1972, 16, 1249-1260. [CrossRef]

4. Gedde, U.W.; Viebke, J.; Leijström, H.; Ifwarson, M. Long-Term Properties of Hot-Water Polyolefin pipes-A Review. Polym. Eng. Sci. 1994, 34, 1773-1787. [CrossRef]

5. Chan, M.; Williams, J. Slow Stable Crack Growth in High Density Polyethylenes. Polymer 1983, 24, 234-244. [CrossRef]

6. Young, R.J.; Beaumont, P.W.R. Failure of Brittle Polymers by Slow Crack Growth. J. Mater. Sci. 1977, 12, 684-692. [CrossRef]

7. Schouwenaars, R.; Jacobo, V.; Ramos, E.; Ortiz, A. Slow Crack Growth and Failure Induced by Manufacturing Defects in HDPE-Tubes. Eng. Fail. Anal. 2007, 14, 1124-1134. [CrossRef]

8. Lu, X.; Qian, R.; McGhie, A.R.; Brown, N. The Effect of Annealing on Slow Crack Growth in an Ethylene-Hexene Copolymer. J. Polym. Sci. Part B Polym. Phys. 1992, 30, 899-906. [CrossRef]

9. Huang, Y.-L.; Brown, N. Dependence of Slow Crack Growth in Polyethylene on Butyl Branch Density: Morphology and Theory. J. Polym. Sci. Part B Polym. Phys. 1991, 29, 129-137. [CrossRef]

10. Huang, Y.-L.; Brown, N. The Effect of Molecular Weight on Slow Crack Growth in Linear Polyethylene Homopolymers. J. Mater. Sci. 1988, 23, 3648-3655. [CrossRef]

11. Pan, Y.; Gao, X.; Wang, Z.; Lei, J.; Shen, K.; Li, Z. Effect of Different Morphologies on Slow Crack Growth of High-Density Polyethylene. RSC Adv. 2015, 5, 28191-28202. [CrossRef]

12. Lu, X.; Zhou, Z.; Brown, N. A Sensitive Mechanical Test for Slow Crack Growth in Polyethylene. Polym. Eng. Sci. 1997, 37, 1896-1900. [CrossRef]

13. Bohm, L.L.; Enderle, H.F.; Fleifßner, M. High-Density Polyethylene Pipe Resins. Adv. Mater. 1992, 4, 234-238. [CrossRef]

14. Gent, A.N.; Gregory, B.L.; Jeong, J.; Charrier, J.-M.; Hamel, F. Effect of a Separator in a Sheet Die on the Anisotropy of Tear Strength of Extruded Medium Density Polyethylene. Polym. Eng. Sci. 1987, 27, 1675-1680. [CrossRef]

15. Capaccio, G.; Rose, L.J.; Kip, B.J. Craze Morphology and Molecular Orientation in the Slow Crack Growth Failure of Polyethylene. J. Appl. Polym. Sci. 2000, 77, 283-296.

16. De Gennes, P.G. Coil-stretch Transition of Dilute Flexible Polymers under Ultrahigh Velocity Gradients. J. Chem. Phys. 1974, 60, 5030-5042. [CrossRef]

17. Somani, R.H.; Yang, L.; Zhu, L.; Hsiao, B.S. Flow-Induced Shish-Kebab Precursor Structures in Entangled Polymer Melts. Polymer 2005, 46, 8587-8623. [CrossRef]

18. Zhou, Q.; Liu, F.; Guo, C.; Fu, Q.; Shen, K.; Zhang, J. Shish-kebab-Like Cylindrulite Structures Resulted from Periodical Shear-Induced Crystallization of Isotactic Polypropylene. Polymer 2011, 52, 2970-2978. [CrossRef] 
19. Jiang, Y.; Mi, D.; Wang, Y.; Wang, T.; Shen, K.; Zhang, J. Insight into Understanding the Influence of Blending Ratio on the Structure and Properties of High-Density Polyethylene/Polystyrene Microfibril Composites Prepared by Vibration Injection Molding. Ind. Eng. Chem. Res. 2019, 58, 1190-1199. [CrossRef]

20. Zhong, G.-J.; Li, L.; Mendes, E.; Byelov, D.; Fu, Q.; Li, Z.-M. Suppression of Skin-Core Structure in Injection-Molded Polymer Parts by in Situ Incorporation of a Microfibrillar Network. Macromolecules 2006, 39, 6771-6775. [CrossRef]

21. Chen, Y.-H.; Zhong, G.-J.; Wang, Y.; Li, Z.-M.; Li, L. Unusual Tuning of Mechanical Properties of Isotactic Polypropylene Using Counteraction of Shear Flow and $\beta$-Nucleating Agent on $\beta$-Form Nucleation. Macromolecules 2009, 42, 4343-4348. [CrossRef]

22. Kech, A.; Ludwig, H.-C.; Möginger, B.; Eyerer, P.; Christiansen, J.D.C. Mechanical Properties of Isotactic Polypropylene with Oriented and Cross-Hatched Lamellae Structure. Int. Polym. Process. 2000, 15, 202-207. [CrossRef]

23. Qin, Y.; Xu, Y.; Zhang, L.; Zheng, G.; Yan, X.; Dai, K.; Liu, C.; Shen, C.; Guo, Z. Interfacial Interaction Enhancement by Shear-Induced $\beta$-Cylindrite in Isotactic polypropylene/Glass Fiber Composites. Polymer 2016, 100, 111-118. [CrossRef]

24. Huang, L.; Wang, Z.; Zheng, G.; Guo, Z.; Dai, K.; Liu, C. Enhancing Oriented Crystals in Injection-Molded HDPE through Introduction of Pre-Shear. Mater. Des. 2015, 78, 12-18. [CrossRef]

25. Mi, D.; Xia, C.; Jin, M.; Wang, F.; Shen, K.; Zhang, J. Quantification of the Effect of Shish-Kebab Structure on the Mechanical Properties of Polypropylene Samples by Controlling Shear Layer Thickness. Macromolecules 2016, 49, 4571-4578. [CrossRef]

26. Mi, D.; La, R.; Wang, T.; Zhang, X.; Zhang, J. Hierarchic Structure and Mechanical Property of Glass Fiber Reinforced Isotactic Polypropylene Composites Molded by Multiflow Vibration Injection Molding. Polym. Compos. 2015, 38, 2707-2717. [CrossRef]

27. Hou, F.; Mi, D.; Zhou, M.; Zhang, J. The Influences of a Novel Shear Layer-Spherulites Layer Alternated Structure on the Mechanical Properties of Injection-Molded Isotactic Polypropylene. Polymer 2017, 122, 12-21. [CrossRef]

28. Gu, X.; Wang, Y.; Jiang, Y.; Liu, M.; Fu, Q.; Zhang, J. High Impact Performance Induced by a Synergistic Effect of Heteroepitaxy and Oriented Layer-Unoriented Layer Alternated Structure in iPP/HDPE Injection Molded Part. Polymer 2019, 175, 206-214. [CrossRef]

29. Liu, M.; Hong, R.; Gu, X.; Fu, Q.; Zhang, J. Remarkably Improved Impact Fracture Toughness of Isotactic Polypropylene via Combining the Effects of Shear Layer-Spherulites Layer Alternated Structure and Thermal Annealing. Ind. Eng. Chem. Res. 2019, 58, 15069-15078. [CrossRef]

Publisher's Note: MDPI stays neutral with regard to jurisdictional claims in published maps and institutional affiliations.

(C) 2020 by the authors. Licensee MDPI, Basel, Switzerland. This article is an open access article distributed under the terms and conditions of the Creative Commons Attribution (CC BY) license (http://creativecommons.org/licenses/by/4.0/). 